Case Report

\title{
A Case of Congenitally Corrected Transposition of the Great Arteries Discovered on Coronary Computed Tomography
}

\author{
Adam T. Marler, Jennifer N. Slim, Travis Batts, James Watts, and Ahmad M. Slim \\ Internal Medicine and Cardiology Service, San Antonio Military Medical Center, 3851 Roger Brooke Drive, Fort Sam Houston, TX \\ 78234-6200, USA \\ Correspondence should be addressed to Jennifer N. Slim; jennifer.slim@amedd.army.mil
}

Received 1 December 2012; Accepted 19 January 2013

Academic Editors: N. Espinola-Zavaleta, Y.-J. Wu, and A. Zirlik

Copyright (C) 2013 Adam T. Marler et al. This is an open access article distributed under the Creative Commons Attribution License, which permits unrestricted use, distribution, and reproduction in any medium, provided the original work is properly cited.

Congenitally corrected transposition of the great arteries is a rare condition accounting for less than $1 \%$ of all congenital cardiac diseases. The fundamental nature of this condition involves a blend of atrioventricular as well as ventriculoarterial discordance. Congenitally corrected transposition of the great arteries is classically associated with three additional abnormalities, including ventricular septal defect, right ventricular outflow tract obstruction, and tricuspid valve abnormalities. Patients with this anomaly have been shown to exhibit reduced exercise tolerance as well as reduced health-related quality of life when compared to patients with normal cardiovascular anatomy. We present the case of a 33-year-old active duty lieutenant in the United States Air Force referred to the cardiology clinic for evaluation of valvular heart disease with subsequent discovery of congenitally corrected transposition of the great arteries on cardiac gated computed tomography.

\section{Case Report}

A 33-year-old male with a history of secundum atrial septal defect percutaneously ended in May 2008 referred for further evaluation of valvular heart disease. Transthoracic echocardiogram completed for a heart murmur was noted to be technically difficult with suboptimal image quality due to mesocardia. Official report from this examination detailed grossly normal left ventricular wall motion with moderate, posteriorly directed, eccentric mitral regurgitation, mild prolapse of the anterior mitral valve leaflet, mild aortic insufficiency, and an echo bright structure contiguous to the lateral aspect of the tricuspid annulus. The right ventricle and right atrium were not well visualized.

At initial visit he reported feeling well overall, but he felt fatigued at 200-400 meters. Exercise stress testing was completed with the patient exercising 12 minutes 26 seconds on a standard Bruce protocol achieving a workload of 14.2 metabolic equivalents (METS) achieving $96 \%$ of maximal age-predicted heart rate with exercise. Exercise testing terminated due to fatigue with the patient reporting no chest pain during evaluation. No ischemic changes were noted on electrocardiogram during the study. Patient's chest X-ray was suggestive of possible RV on the left side of the chest with possible dextrocardia or ccTGA in the differential (Figure 1).

Subsequently, the patient underwent imaging evaluation with cardiac computed tomography (CT) for further evaluation of cardiac structure. CT angiography of the coronary arteries revealed normal origin and course of all coronary vessels with no evidence of coronary artery disease. CT evaluation of cardiac structure showed the right ventricle receiving blood from the left atrium through the tricuspid valve and the left ventricle receiving blood from the right atrium through the mitral valve. The right ventricle was shown to eject blood into the aorta with the left ventricle pumping blood into the pulmonary artery. The right ventricle demonstrated concentric hypertrophy measuring $9.9 \mathrm{~mm}$ at the mid-interventricular septum. Findings were noted to be consistent with a congenitally corrected L-transposition of the great arteries (ccTGA) (Figure 2).

Following imaging evaluation, the patient was diagnosed with systemic hypertension and begun on hydrochlorothiazide for initial therapy. Antihypertensive therapy was changed from hydrochlorothiazide to lisinopril during a brief admission for atypical chest pain due to failure to reach treatment goals with the diuretic. Mandatory military 


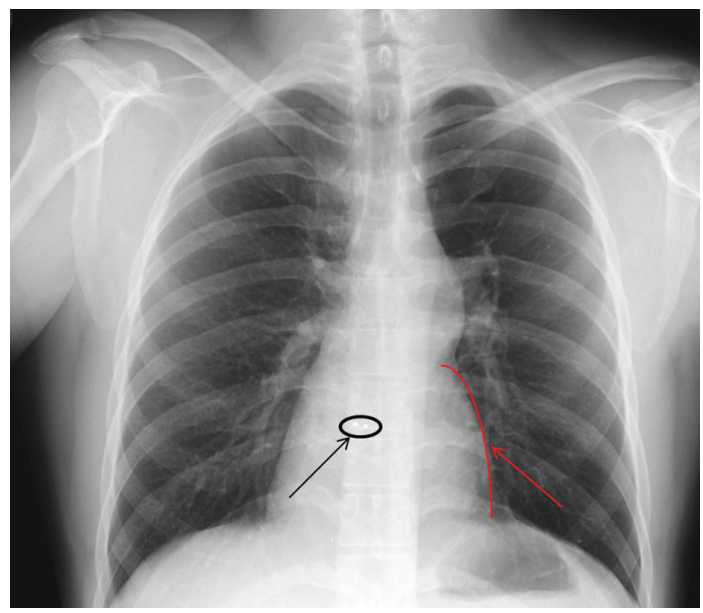

FIGURE 1: X-ray changes anticipated in ccTGA with right ventricular border outlined with red arrow and Amplatzer occluder device outlined with dark arrow.

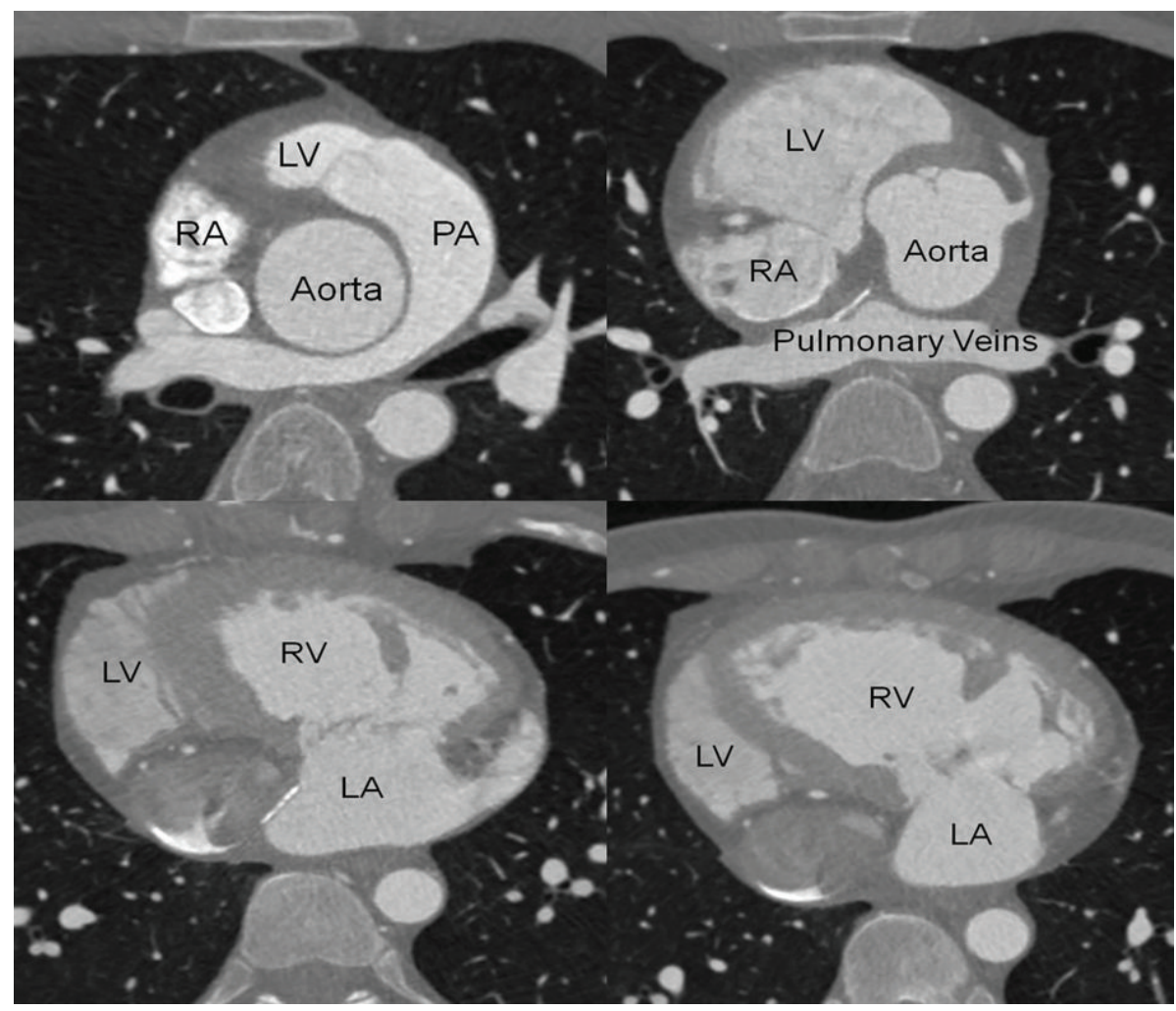

FIGURE 2: Anatomical confirmation of ccTGA on coronary CT (CTA).

Medical Evaluation Board (MEB) was initiated due to the presence of moderate valvular disease and, as part of this process, cardiopulmonary exercise testing was scheduled despite recent history of gated exercise testing and no worsening of daily function per patient report. Cardiopulmonary exercise testing was carried out using cycle ramp protocol beginning at 0 Watts and incrementally increasing to workload of 157 Watts. The patient exercised for 7 minutes 10 seconds with testing discontinued due to leg fatigue. Peak $\mathrm{VO}_{2}$ measured at $29.7 \mathrm{~mL} / \mathrm{kg} / \mathrm{min}$ representing $8.5 \mathrm{METS}$ or $77 \%$ of maximum predicted. Anaerobic threshold occurred at 51\% of measured $\mathrm{VO}_{2}$ max. Peak heart rate was 180 beats per minute with a blood pressure peak of 146/70. There was delayed heart rate recovery with noted heart rate of 150 beats per minute at three minutes of recovery. Peak respiratory rate was 42 per minute with $\mathrm{VE}$ of $68 \mathrm{~L} / \mathrm{min}$ and $\mathrm{VE} / \mathrm{VCO}_{2}$ slope of 29.9 and pulse oximeter reading of $>95 \%$. Overall capacity of the patient has dropped markedly as compared to the 14.2 METS achieved on a standard exercise stress test performed a year prior. 


\section{Discussion}

Congenitally corrected transposition of the great arteries (ccTGA) accounts for less than $1 \%$ of all congenital cardiac anomalies [1]. Most patients with ccTGA have at least one additional cardiac abnormality consisting of pulmonary stenosis (41\%), ventricular septal defects (37\%), and atrial septal defects (19\%) as well as Ebstein's anomaly of the tricuspid valve (7\%) [2]. In our patient, atrial septal defect was detected and an occlude device was placed but yet the diagnosis of ccTGA was never contemplated till a chest Xray was obtained followed by coronary CT for confirmation. The utility of coronary CT (CTA) is increasing in screening military service members for the workup of acute symptoms of chest pain; however, this case proves that screening CTA for military recruits with any concern of congenital disorder might be warranted with excellent accuracy when Transthoracic echocardiography proves to be limited.

In a retrospective study of 41 patients with ccTGA who had completed lung function testing and cardiopulmonary exercise testing, aerobic capacity in patients with ccTGA is severely diminished with $\mathrm{VO}_{2}$ max ranged from 11 to $22 \mathrm{~mL} / \mathrm{kg} / \mathrm{min}$ that corresponds to $30-50 \%$ of results achieved by healthy patients [3]. In the same retrospective study, systemic RV ejection fraction rose by only $2 \%$ in ccTGA patients, while pulmonary LV ejection fraction reduced by $2 \%$ with exertion. Patients on ACE inhibitors had significantly lower pulmonary LV ejection fraction at max exertion compared to those not on ACE inhibitors [3]. These findings might explain the acute reduction in exercise capacity in our patient immediately after initiation of ACI for blood pressure control.

\section{Conclusion}

Congenitally corrected transposition of the great arteries is a rare cardiac anomaly where many patients will remain asymptomatic for much of their lives. ccTGA patients have a reduced tolerance for exercise and have reported reduced health-related quality of life compared to a control population. As noted in this patient, ACE inhibitors may intensify exercise intolerance in these patients.

\section{Disclaimer}

The opinions or assertions contained herein are the private views of the authors and are not to be construed as reflecting the views of the Department of the Army or the Department of Defense.

\section{References}

[1] C. A. Warnes, "Transposition of the great arteries," Circulation, vol. 114, no. 24, pp. 2699-2709, 2006.

[2] E. L. W. Tay, A. Frogoudaki, R. Inuzuka et al., "Exercise intolerance in patients with congenitally corrected transposition of the great arteries relates to right ventricular filling pressures," International Journal of Cardiology, vol. 147, no. 2, pp. 219-223, 2011.
[3] P. M. Fredriksen, A. Chen, G. Veldtman, S. Hechter, J. Therrien, and G. Webb, "Exercise capacity in adult patients with congenitally corrected transposition of the great arteries," Heart, vol. 85, no. 2, pp. 191-195, 2001. 


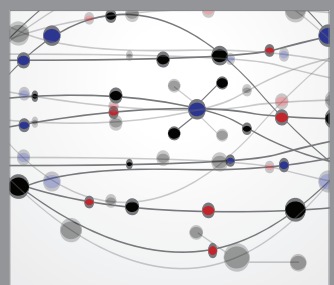

The Scientific World Journal
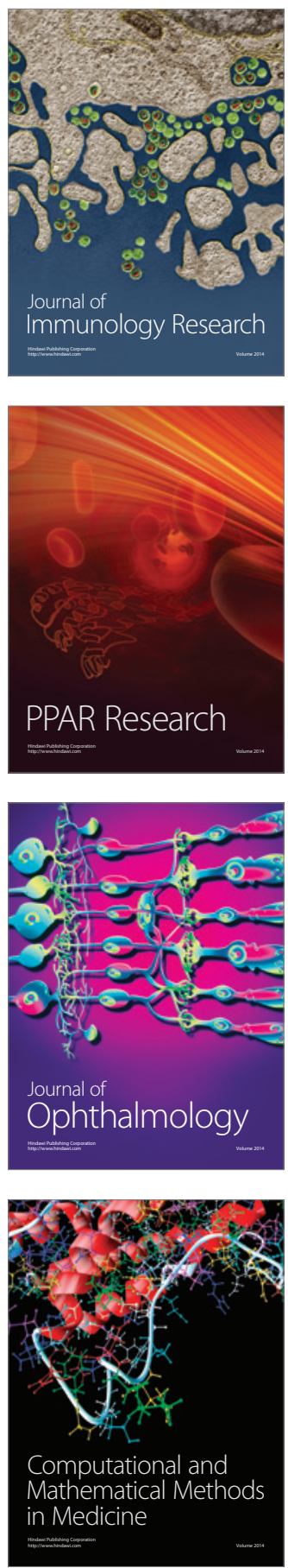

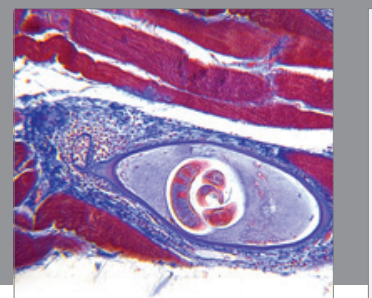

Gastroenterology

Research and Practice
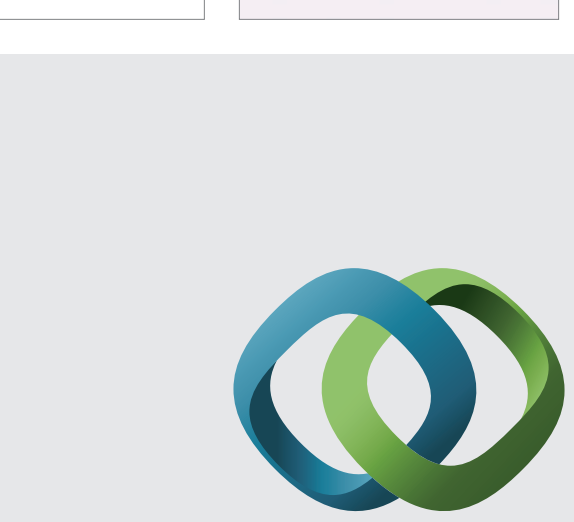

\section{Hindawi}

Submit your manuscripts at

http://www.hindawi.com
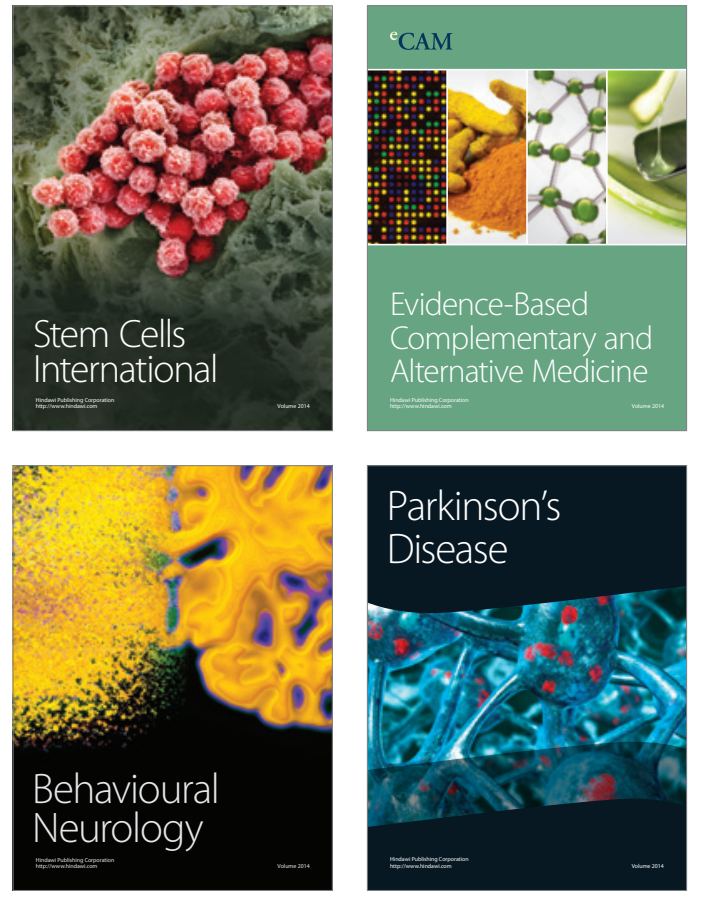
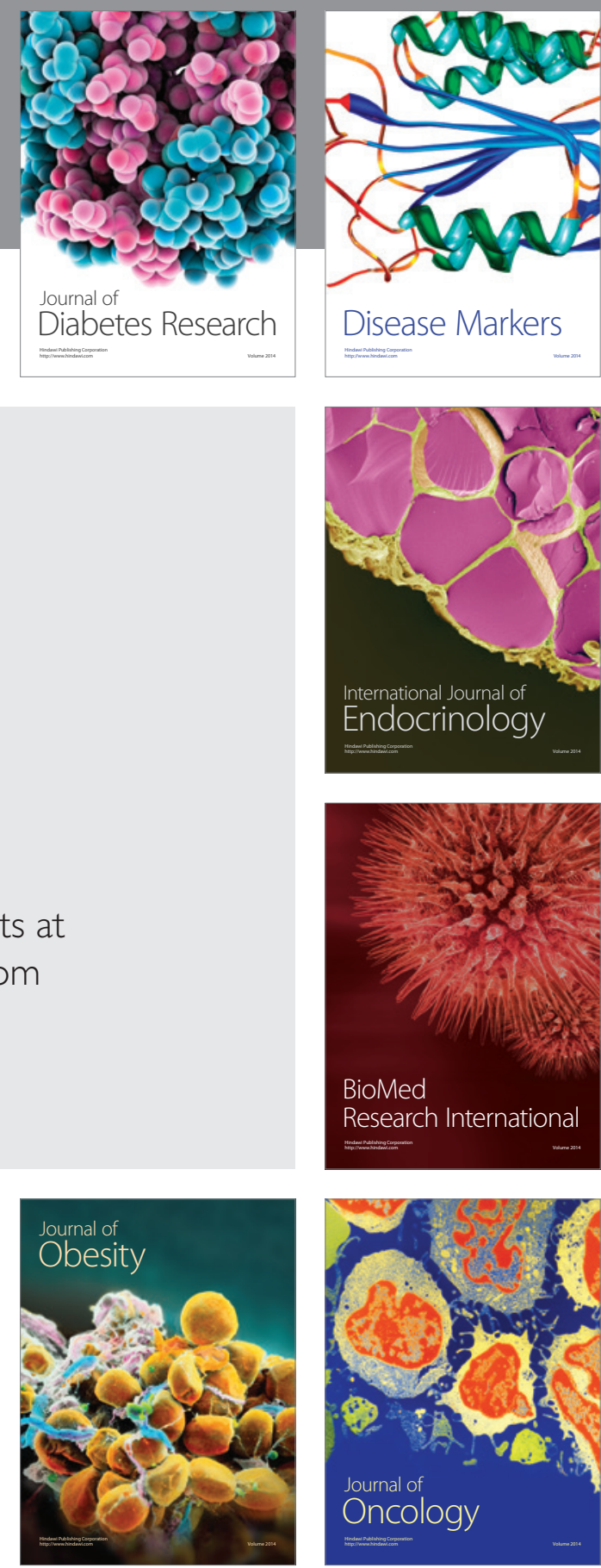

Disease Markers
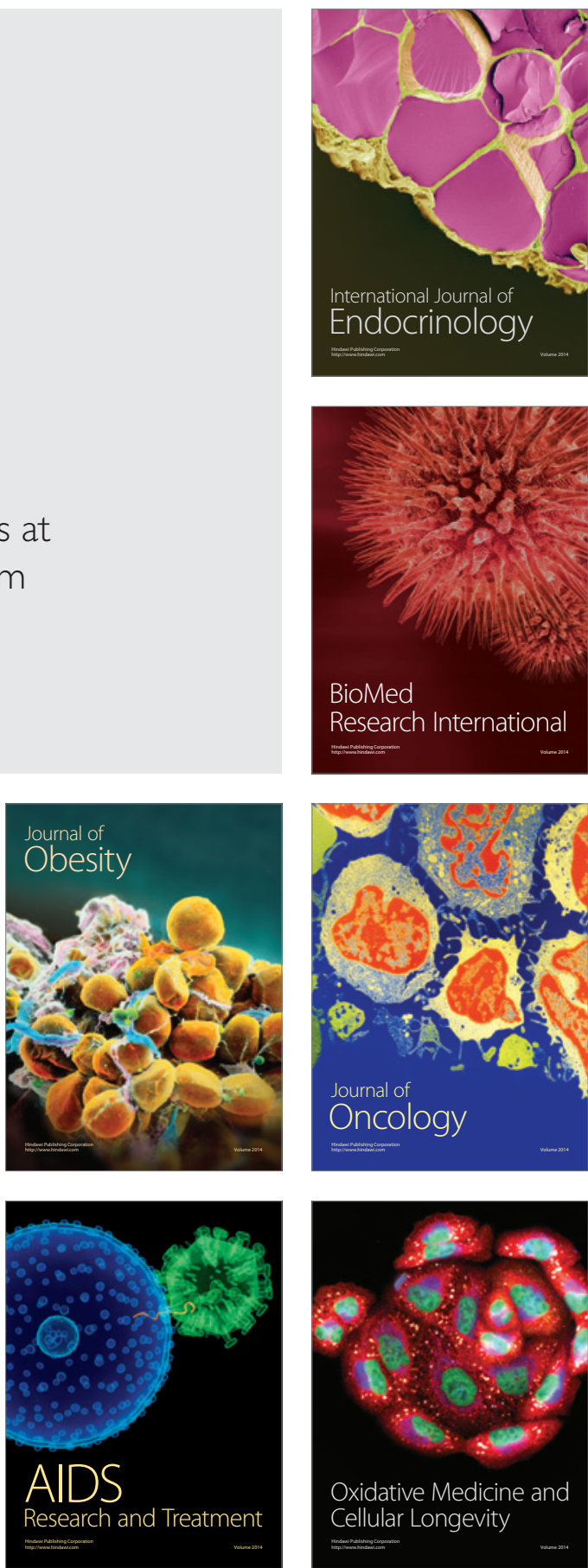